\title{
Laparoscopic Management for Small Bowel Obstruction: Experience From a UK District General Hospital With One-Year Follow-Up
}

\author{
Bhavani Mothe ${ }^{\mathrm{a}, \mathrm{b}}$, Usman Khan ${ }^{\mathrm{a}}$, Chris Smart ${ }^{\mathrm{a}}$
}

\begin{abstract}
Background: Small bowel obstruction (SBO) is a common surgical emergency usually treated via standard laparotomy. But with the advancement of laparoscopic surgical skills, more surgeons are undertaking laparoscopic approach as well to treat uncomplicated SBO. We aimed to compare outcomes following laparoscopic vs. open surgical techniques in a district general hospital.
\end{abstract}

Methods: A retrospective review of case notes of patients who underwent operative surgical treatment for SBO between January 2012 and July 2018 was carried out. Mann-Whitney U test was used for nominal data and Chi-square test for categorical data analysis.

Results: A total of 99 surgical procedures were carried out for SBO during this period at our district general hospital with 31 laparoscopic and 68 open procedures. Fifteen out of 31 were completed laparoscopically (totally laparoscopic approach (TLA)), while 16/31 were converted to open procedure (laparoscopic-assisted group (LAG)) to complete the operation. Median age for open group was 69 years (26 - 91 years) vs. 71 years (23 - 94 years) for laparoscopic group. Median length of stay was 10 vs. 6.5 days for single band adhesions treated laparoscopically $(\mathrm{P}=0.02)$. Median Charlson comorbidity index was 6 vs. 5 (TLA). Eight out of 31 in laparoscopic group had complications, whereas $27 / 68$ in open group $(\mathrm{P}=0.26)$. The 30 -day mortality was $5 / 68$ for open vs. none for laparoscopy group. The 1-year follow-up for all patients confirmed 7/68 in open vs. 1/31 laparoscopic group mortalities.

Conclusion: Laparoscopic surgical approach for SBO seems to have statistically significant lower small bowel resection rate and length of stay in this hospital when compared to laparotomy in selected patients.

Keywords: Adhesions; Bowel obstruction; Small intestine; Laparoscopy; Length of stay

Manuscript submitted November 9, 2020, accepted November 19, 2020

Published online January 30, 2020

aDepartment of General and Colorectal Surgery, Macclesfield District General Hospital, Macclesfield, East Cheshire NHST Trust, Victoria Road, Macclesfield, SK10 3BL, UK

${ }^{b}$ Corresponding Author: Bhavani Mothe, Department of Surgery, Macclesfield General Hospital, Macclesfield, SK10 3BL, UK. Email: b.mothe@nhs.net

doi: https://doi.org/10.14740/jcs430

\section{Introduction}

Increasing scrutiny of emergency general surgery lead to the National Emergency Laparotomy Audit (NELA) with a major focus on improving patient outcomes by improving the quality of care that was being delivered [1]. Small bowel obstruction (SBO) is a common surgical emergency which is leading to 12,000 laparotomies in the UK [2]. The leading cause for intestinal obstruction in $60-80 \%$ of the cases is adhesions from previous surgery and up to $50 \%$ of these patients require surgical treatment [3]. Because of the recurring nature of this surgical problem, there is a significant long-term impact on healthcare costs and morbidity [4]. Although the standard approach is laparotomy, many studies have demonstrated the benefit of laparoscopic surgical approach in reducing the morbidity and mortality $[3,5]$.

Even though NELA provided rich data on patients who underwent surgery, it failed to capture information on patients who are managed conservatively. To overcome this deficit in information, the UK Royal colleges along with professional specialty associations helped the trainee research collaborative to deliver the National Audit of Small Bowel Obstruction [6, 7]. Historically there has been reluctance to use laparoscopy in acute SBO because of associated difficulties such as working in reduced space due to distended bowel and associated higher risk of iatrogenic bowel injury. Increasing training and advances in laparoscopic skills is shifting the surgical management attitude towards laparoscopy more frequently $[5,8$ 11]. The World Society of Emergency Surgery Adhesive SBO Working Group deems pneumoperitoneum related factors such as hemodynamic instability or cardiopulmonary insufficiency as absolute contraindications for laparoscopic treatment of SBO [12, 13]. Several benefits have been demonstrated by using laparoscopic approach in the management of SBO and these include reduced hospital stay, decreased postoperative pain and lower morbidity overall $[14,15]$. Through this retrospective study, we aim to report the impact of laparoscopic approach on patient outcomes in a district general hospital following SBO with 1-year follow-up.

\section{Materials and Methods}

This was a case series of SBO from a UK district general hospital from January 2012 to July 2018. During this time, a to- 
Table 1. Demographics of Patients

\begin{tabular}{llll}
\hline SBO $(\mathbf{N}=\mathbf{9 9})$ & Laparoscopic $(\mathbf{N}=\mathbf{3 1})$ & Open (laparotomy) $(\mathbf{N}=\mathbf{6 8})$ & P value \\
\hline Age (years) & $71(23-94)$ & $69(26-91)$ & 6.016 \\
Charlson comorbidity index (median) & TLA: $3(0-12)$ & $6.12)$ & 0.23 \\
& LAG: $5(2-11)$ & & \\
& All laparoscopic data: $6(0-12)$ & A\&E: 60 \\
Route of referral & A\&E: 24 & Others: 6 & GP: 2 \\
& Others: 5 & $35 / 68(51 \%)$ \\
\hline History of prior abdominal surgery & GP: 2 & & \\
\hline
\end{tabular}

SBO: small bowel obstruction; TLA: totally laparoscopic approach; LAG: laparoscopic-assisted group; A\&E: accident and emergency department; GP: general practitioners.

tal of 118 procedures were recorded for SBO, out of which 19 procedures were excluded. Reasons for exclusion from the study include patients who had open and close laparotomies for conditions like complete bowel ischemia $(n=5)$, frozen abdomen due to advanced malignancy/adhesions $(n=7)$ and patients who had incomplete set of notes $(n=3)$ or lacking in follow-up data $(n=4)$. A comprehensive review of clinical notes of the patients was undertaken and the collected information was further cross referenced by confirming with respective general practitioners about re-admissions in the 12 months follow-up period after the index procedure. In some cases, patients were contacted directly to confirm whether readmission was recorded during the follow-up period.

The hospital audit and research department was contacted for study approval. This study was conducted in compliance with the ethical standards of East Cheshire NHS Trust on human subjects as well as with the Helsinki Declaration. Data analysis was carried out for statistical significance between laparoscopic vs. open group by using Mann-Whitney U test for nominal data and Chi-squared test for categorical data.

\section{Results}

The details of the patients are presented in Table 1. The me- dian age of patients who were treated for SBO was 71 years (range 23 - 94) in laparoscopic group and 69 years (range 26 - 91) in open group. Most of the patients (87\%) were referred from accident and emergency department, while 9\% from other specialities within the hospital and only $4 \%$ from the general practitioners. Patients who were already in the hospital under different clinical teams experienced delay in diagnosis and treatment of the SBO. The study group has 68 procedures completed using open laparotomy method, whereas the laparoscopic group was 31 , out of which $15 / 31$ procedures were completed through totally laparoscopic approach (TLA), whereas $16 / 31$ needed extension of the umbilical wound (laparoscopicassisted group (LAG)) to allow completion of the procedure due to multiple adhesions or technical difficulty or need for bowel resection.

Preoperative characteristics of the study group are represented in Table 2. This includes radiological assessment for confirmation of the etiology of SBO and planning management accordingly. All patients in this cohort had computed tomography (CT) scan before definitive plan for surgery was made. Patients also received nasogastric decompression (NG) for relieving the pressure from the obstructed bowel (NG) and urinary catheter insertion along with intravenous fluid replacement for maintaining the fluid balance. Acute inflammatory process has been noted to be present in patients with SBO

Table 2. Preoperative Characteristics

\begin{tabular}{llll}
\hline SBO $(\mathbf{N}=\mathbf{9 9 )}$ & Laparoscopic $(\mathbf{N}=\mathbf{3 1})$ & Open (laparotomy) $(\mathbf{N}=\mathbf{6 8})$ & $\begin{array}{l}\text { P value (Mann- } \\
\text { Whitney U test) }\end{array}$ \\
\hline CRP & TLA: $8(1-34)$ & $21.5(1-433)$ & 0.03 \\
& LAG: $13.5(1-212)$ & & 0.05 \\
& All laparoscopic data: $8(1-212)$ & $10.5(4.4-29.9)$ & 0.05 \\
WBC & TLA: $9(5-26)$ & & 0.32 \\
& LAG: $9(4-22)$ & & 0.06 \\
& All laparoscopic data: $9(4-26)$ & $1(0.5-15)$ & 0.09 \\
Time to operation from & TLA only: $1(0.5-1.0)$ & Mean: 2.55 days & 0.005 \\
admission (days) & LAG: $1.5(0.5-9)$ & 0.27 \\
& All laparoscopic data: $1(0.5-9)$ & & 0.21 \\
\hline
\end{tabular}

SBO: small bowel obstruction; CRP: C-reactive protein; WBC: white blood cell count; TLA: totally laparoscopic approach; LAG: laparoscopic-assisted group. 


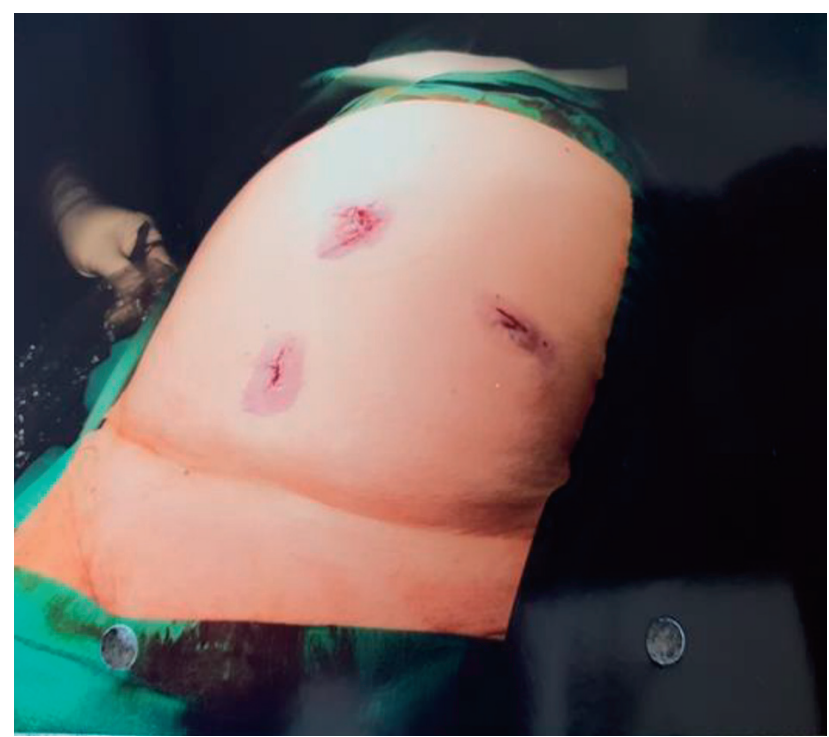

Figure 1. Laparoscopic location of port sites.

when the preoperative C-reactive protein (CRP) and white blood cell count (WBC) were recorded. Increased inflammation can cause decreased blood supply to the small bowel with risk of strangulation and ischemia $[16,17]$.

In this study, the laparoscopic cohort overall had $25 \%$ (8/31) of the patients with CRP over 30 with TLA having $13 \%$ $(2 / 15)$ and LAG having $37 \%(6 / 16)$. Similarly, the WBC greater than $12 \times 100 / \mathrm{mm}^{3}$ was found in $22.5 \%(7 / 31)$ of the patients with 4/15 (26\%) for TLA group and 18\% (3/16) for LAG. The laparotomy cohort had $45 \%(31 / 68)$ of the patients with CRP over 30 and $\mathrm{WBC}>12$ in $36 \%(25 / 68)$ of the patients.
Adhesions is known to be the most important single etiological factor for causing SBO [1-3] and having previous surgery increases the risk of adhesions formation. In this study, we have noted in laparotomy group $45 \%$ (31/68) of the patients had previous abdominal/pelvic surgery, whereas in the laparoscopic group, it was 58\% (18/31) of the patients who had previous surgical intervention. The median time to surgery (Table 2) from admission was $24 \mathrm{~h}$ (1 day) for the whole laparoscopic group; however, it was 1.5 days for LAG (range $0.5-9)$. In the laparotomy group, although the mean time to surgery was $58 \mathrm{~h}$ (2.55 days), the median was still 1 day (range $0.5-15)$.

The laparoscopic technique involved achieving pneumoperitoneum either through modified Hasson technique [18] with one 12-mm umbilical port or Veress needle insertion in the left upper quadrant. Further two 5-mm working ports were employed in $90 \%$ of the cases to complete the procedure (Fig. 1). In the rest of the cases, one or two additional 5-mm ports were used to complete the procedure. In laparoscopic assisted cases, the umbilical port was usually extended to allow further dissection or resection of the small bowel.

In the TLA group $(\mathrm{n}=15), 10$ patients had single band adhesion (Fig. 2) as cause of obstruction, whereas two patients had multiple adhesions and the rest included two internal hernias and one foreign body. The causes for obstruction in the LAG included 2/16 single band adhesion, 11/16 multiple adhesions and 3/16 other causes (lymphoma and hernias). Overall, in this group $35.4 \%(11 / 31)$ of the patients did not have prior surgery with $10 / 15(66.6 \%)$ of them from the TLA and $12 \%(2 / 16)$ in the LAG (Table 3$)$. This compares well with $48 \%(33 / 68)$ of the patients in the laparotomy group. Fifty percent $(34 / 68)$ of the patients in laparotomy group had single

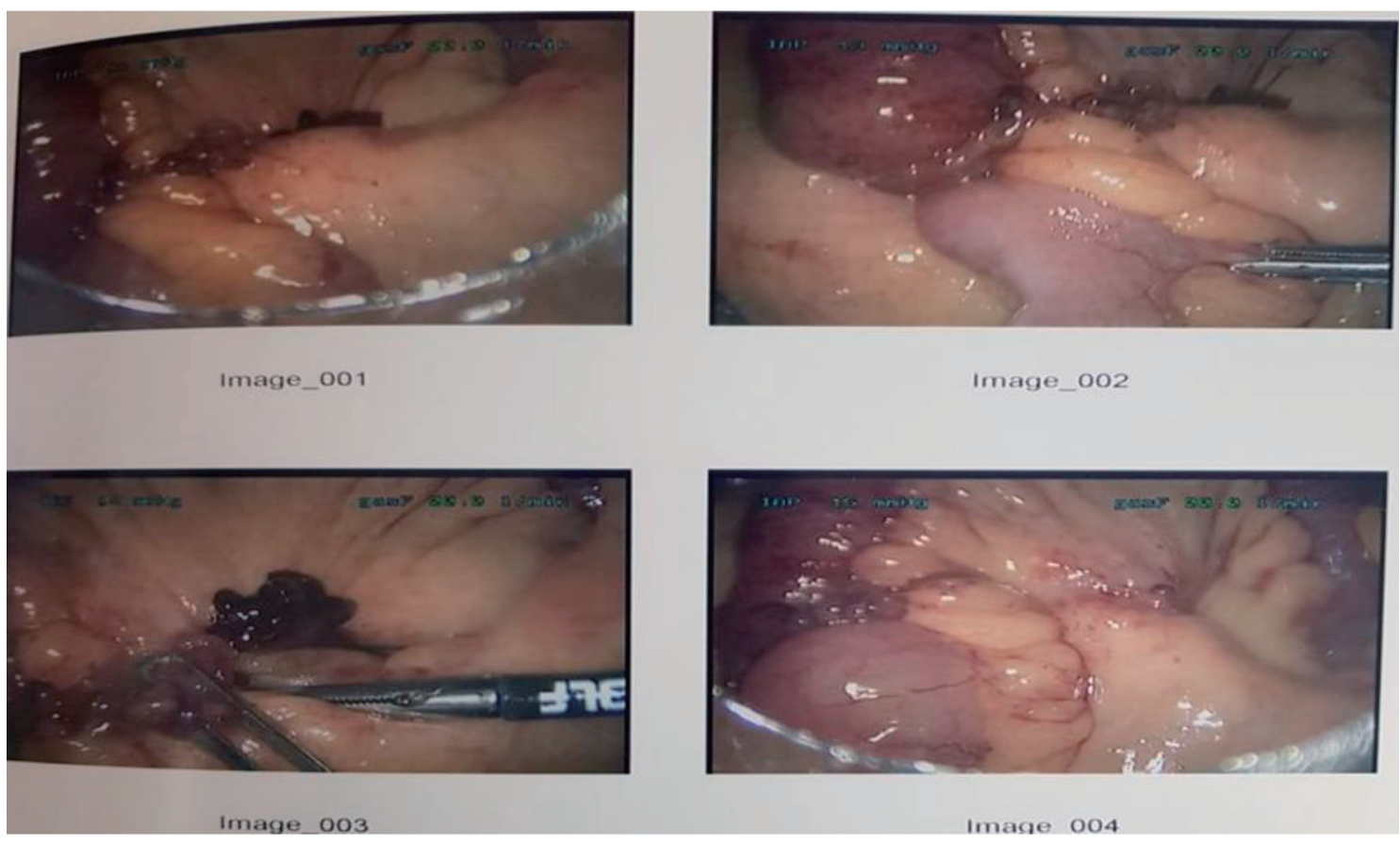

Figure 2. Band adhesion causing obstruction demonstrated in images 1 to 3 and released in image 4 . 
Table 3. Causes of SBO Confirmed Intraoperatively

\begin{tabular}{ll}
\hline Laparoscopic surgery group $(\mathbf{N}=\mathbf{3 1})$ & Laparotomy group $(\mathbf{N}=\mathbf{6 8})$ \\
\hline TLA: 10 single band adhesion, 2 multiple adhesions, 3 others (hernias/foreign body) & Single band adhesion: 34 \\
LAG: 2 single band adhesion, 11 multiple adhesions, 3 & Multiple adhesions: 21 \\
others (hernias, small bowel lymphoma) & Others (tumors/ischemia/hernias): 13 \\
\hline
\end{tabular}

SBO: small bowel obstruction; TLA: totally laparoscopic approach; LAG: laparoscopic-assisted group.

Table 4. Single Band Adhesion: Sub-Group Analysis

\begin{tabular}{llll}
\hline SBO $(\mathbf{N}=\mathbf{4 8})$ & Laparoscopic $(\mathbf{N}=\mathbf{1 2})$ & Open (laparotomy) $(\mathbf{N}=\mathbf{3 4})$ & P value (Mann-Whitney U test) \\
\hline Age (years) & $69.5(35-90)$ & $75(46-89)$ & 0.37 \\
Charlson comorbidity index (median) & $3(2-12)$ & $5.5(2-11)$ & 0.16 \\
History of prior abdominal surgery & $4 / 12(33 \%)$ & $17 / 34(50 \%)$ & 0.40 \\
Small bowel resection rate & None & 10 & 0.06 \\
HDU/ITU care & $2(16.6 \%)$ & $10(29.4 \%)$ & 0.52 \\
Complication AKI & $3(25 \%)$ & $9(26 \%)$ & 0.83 \\
Overall complication rate & $4(33 \%)$ & $10(29 \%)$ & 0.84 \\
Length of hospital stay (median days) & $6.5(2-28)$ & $10(3-31)$ & 0.02 \\
\hline
\end{tabular}

SBO: small bowel obstruction; HDU: high dependency unit; ITU: intensive therapy unit; AKI: acute kidney injury.

band adhesion as intraoperative cause for obstruction, whereas multiple adhesions contributed 31\% (21/68) with other etiology $11 \%$ (8/68 hernias/pelvic mass/ischemia) and tumors $7 \%$ $(5 / 68)$.

In this study, intraoperatively the laparoscopic group had $16 \%(5 / 31)$ bowel resection with only one patient (1/15) needing it from the TLA, whereas in the laparotomy group, the small bowel resection rate was 40\% (27/68). Almost all patients who had bowel resection went to high dependency unit postoperatively for monitoring. Ninety-eight percent of the study participants (30/31 and 67/68) had their nutritional status assessed during the acute admission performed through MUST score [19]. Two out of 31 patients $(6 \%)$ in the LAG needed parenteral nutritional support compared to $16 / 68(23.5 \%)$ in the laparotomy group.

Sub-group analysis of single band adhesion patients (Table 4) between both laparoscopic and open groups shows that the age distribution is reflective of the main study. The laparoscopic group had less comorbid patients compared to the open group and the history of previous surgery was marginally reduced than the open group (33\% vs. 50\%). Similarly, the rate of small bowel resection was far reduced compared to open group (none vs. 10) but fails to reach statistical significance $(\mathrm{P}<0.06)$. The rate of admission to high dependency unit or critical care unit was nearly half of the open group (16.6\% vs. $29.4 \%$ ) with statistically significant reduced stay in hospital (6.5 vs. 10 days) when patients are treated through laparoscopic approach $(\mathrm{P}<0.02)$.

Table 5. Postoperative Characteristics

\begin{tabular}{llll}
\hline SBO $(\mathbf{N}=\mathbf{9 9 )}$ & Laparoscopic $(\mathbf{N}=\mathbf{3 1})$ & Open (laparotomy) $\mathbf{N}=\mathbf{6 8})$ & $\begin{array}{l}\text { P value with Yates' correc- } \\
\text { tion (Chi-squared test) }\end{array}$ \\
\hline Small bowel resection rate & $5 / 31(16 \%)$ & $27 / 68(39 \%)$ & 0.03 \\
HDU/ITU care & $8 / 31(25 \%)$ & $28 / 68(41 \%)$ & 0.21 \\
Complication AKI & $6 / 31(19 \%)$ & $19 / 68(27.9 \%)$ & 0.50 \\
Overall complication rate & $8 / 31(25 \%)$ & $27 / 68(39 \%)$ & 0.26 \\
& Chest infection: 2 & Chest infection: 12 & 0.025 (Mann-Whitney U test) \\
Length of Hospital & Wound infection: 1 & Wound infection: 10 & 0.32 \\
Stay (Median days) & TLA: $5(2-28)$ & $11(4-140)$ & 0.025 \\
LAG: $11.5(4-19)$ & & 0.12 \\
\hline 30-day mortality & All lap: $10(2-28)$ & $4 / 68$ & 0.13 \\
\hline 1-year mortality & 0/31 & $7 / 68$ & \\
\hline
\end{tabular}

SBO: small bowel obstruction; HDU: high dependency unit; ITU: intensive therapy unit; TLA: totally laparoscopic approach; LAG: laparoscopicassisted group; AKI: acute kidney injury. 
Table 6. Comparison Between Similar Studies

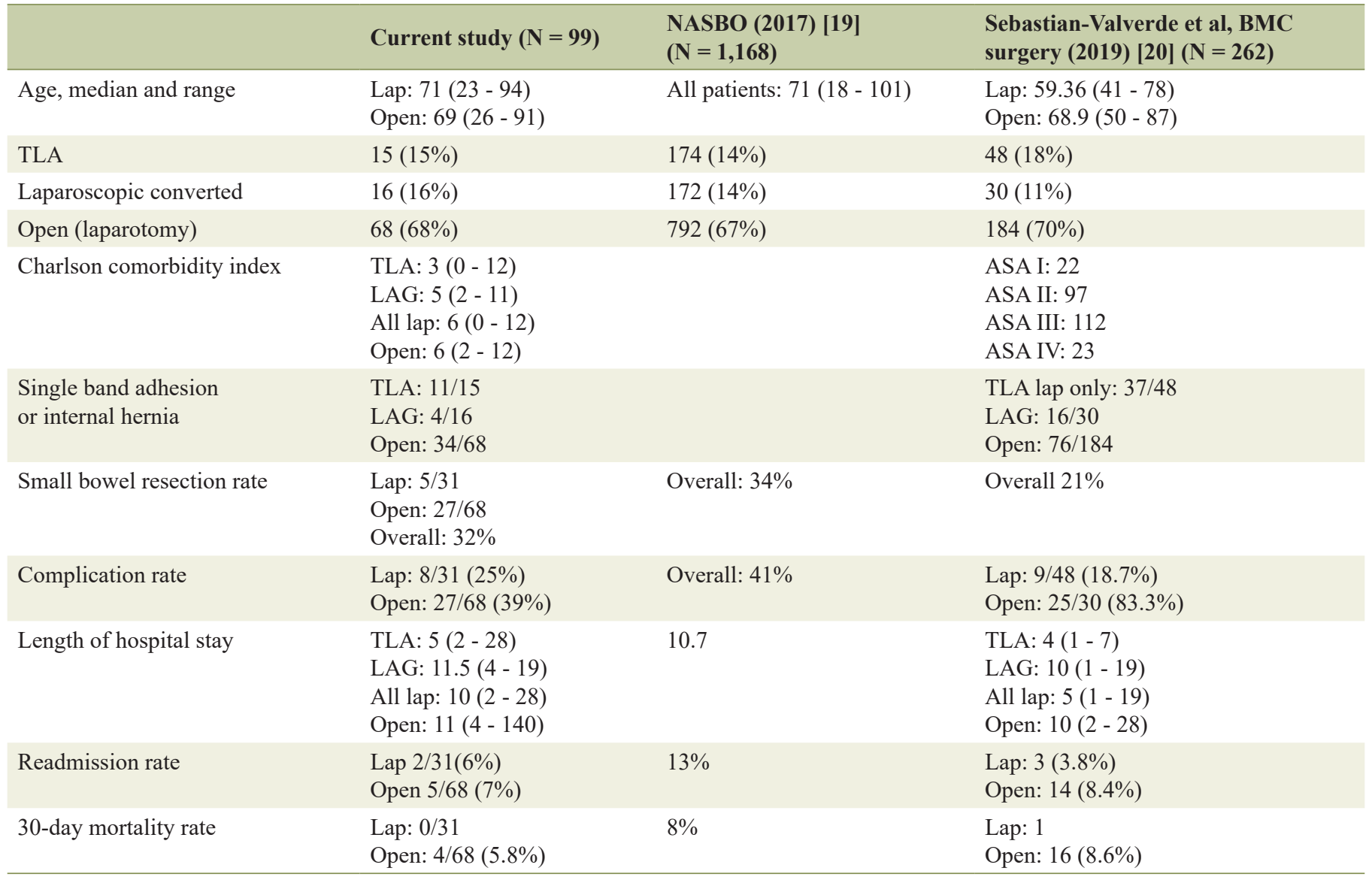

TLA: totally laparoscopic approach; LAG: laparoscopic-assisted group.

\section{Postoperative complications}

In the TLA group, only $1 / 15$ needed to go back to theater for further surgery, as SBO did not resolve completely after laparoscopic band adhesion release and patient needed laparotomy to free more adhesions. In comparison, 3/68 in the laparotomy group needed further surgery due to complete wound dehiscence $(n=1)$, further small bowel ischemia requiring further resection $(\mathrm{n}=1)$ and non-resolving ileus after band adhesions release $(n=1)$.

All major postoperative complications are recorded in Table 4 and these consisted mainly of acute kidney injury (AKI) $(6 / 31(19 \%)$ in laparoscopic group and 19/68 (28\%) in laparotomy group), chest infection (2/31 vs. 12/68) and wound infection (1/31 vs. 10/68). The median length of hospital stay varied significantly between the groups. For the TLA, it was 5 days (range 2 - 28) and for LAG it was 11.5 days (range 4 - 19). The laparotomy group had almost similar length of hospital stay as LAG at 11 days (range 4 - 140). The 90-day readmission rate was 2/31 (6\%) for laparoscopic group and 5/68 (7\%) for open group and none of them needed further surgery.

There was no mortality recorded in the laparoscopic group at 30 days, whereas in the laparotomy group, there were $4 / 68$ $(5.8 \%)$ recorded deaths. After 1-year follow-up of these pa- tients, mortality was noted to be $1 / 31(0.03 \%)$ in laparoscopic group and $7 / 68(10 \%)$ in the laparotomy group and all patients died due to non-bowel related cause.

\section{Discussion}

Our study highlights that patients presenting with SBO when treated with laparoscopic approach seem to be associated with better postoperative outcomes including reduced hospital stay than open approach (Tables 4 and 5). This study closely represents the National Audit of Small Bowel Obstruction of United Kingdom data from 2017 (NASBO) [19] and similarly study conducted by Sebastian-Valverde et al [20] showed comparable results with our study (Table 6).

Based on the type of previous surgical intervention (laparoscopic vs. laparotomy) and the number of procedures performed, the pathogenesis of future adhesions and the nature of SBO seem to vary [21]. Simple paraumbilical/umbilical hernias and appendix operations seem to cause lesser adhesions when compared to long midline laparotomy wherein denser adhesions are noted which increases the future need for further surgery [22]. Effectively previous laparotomy seems to be a predictive risk factor for developing future SBO 
and need for further surgery when compared to laparoscopic approach [23-25] (odds ratio $(\mathrm{OR})=0.8 ; \mathrm{P}<0.001)$. In this study, almost all laparoscopic converted or assisted group had previous abdominal surgery compared to TLA and open group (Table 1: $87 \%$ vs. $40 \%$ vs. $51 \%$ ). The higher rate of conversion in laparoscopic group is probably because of keeping low threshold for accepting Bologna guidelines for SBO management $[12,13]$.

In their systematic review, Wiggins et al $[14,25]$ reported overall reduction in postoperative morbidity following laparoscopic approach $(\mathrm{OR}=0.34 ; \mathrm{P}<0.0001)$ which can vary between $4 \%$ and $40 \%$. Since there can be variation in the way Clavien-Dindo classification can estimate complications, we have considered in our study only the major complications which can influence the patient length of stay considerably such as chest/wound infection and AKI. In our study, small bowel resection rate was much less in laparoscopic group whether it is for single band adhesion SBO (Table 4: none vs. $10, \mathrm{P}<0.06$ ) or other etiology (Table 5: $16 \%$ vs. $39 \%, \mathrm{P}<$ $0.03)$ compared to laparotomy. The overall complication rate was $25 \%$ vs. $39 \%(\mathrm{P}=0.26)$ which is reduced rate for laparoscopic group leading to shorter stay of 5 days in TLA group vs. 11 days for laparotomy group (Table 5; $\mathrm{P}=0.025$ ). Similar result with statistically significant difference in hospital stay can also be found in single band adhesion treated with laparoscopic means (Table 4: 6.5 vs. 10 days; $P=0.02$ ) [26].

The laparoscopic approach shows better postoperative outcomes even when the conversion rate is $16 \%$ which is higher than the national average of $14 \%$ (NASBO [19]) and $11 \%$ in similar other study [20]. Maybe the decision to operate early in laparoscopic group (Table 2; 24 h vs. $58 \mathrm{~h}, \mathrm{P}=0.005$ ) with lower inflammatory profile (CRP 8 vs. $21, \mathrm{P}=0.03)$ is producing lesser complications postoperatively and hence contributing to early discharge of patients from the hospital [11, 14]. The 90-day readmission ratio is almost similar between the groups $(2 / 31,6.45 \%$ vs. $7.35 \%, 5 / 68)$ with no discernible contributing factors with the 30-day and 1-year follow-up data showing no significant differences in the mortality rates (Table 5).

The main reasons for conversion in cases started laparoscopically in our study are technically challenging adhesions, need for bowel resection and reducing the risk of enterotomy during the procedure. Selection bias may have been introduced by choosing to operate early on lesser comorbid patients (Table 1: Charlson comorbidity index 3 vs. $6 ; \mathrm{P}=0.016$ ) leading to decreased complications and hospital stay. Larger prospective randomized trials like Sallinen et al [26] are needed to reinforce the use of laparoscopic surgery routinely in SBO management to improve postoperative outcomes for the patients.

\section{Conclusion}

With the help of this study, we strongly advocate the routine and early use of laparoscopy surgery in the management of $\mathrm{SBO}$ even in a district general hospital setting. Compared to laparotomy, laparoscopic approach seems to be associated with better postoperative outcomes and shorter stay in the hospital, especially in selected patients.

\section{Acknowledgments}

None to declare.

\section{Financial Disclosure}

There was no specific funding source to be mentioned.

\section{Conflict of Interest}

The authors declare that they have no conflict of interest.

\section{Informed Consent}

All subjects provided written informed consent.

\section{Author Contributions}

BM designed and performed the study and drafted the manuscript. UK and CS did critical editing and carefully supervised this manuscript preparation and writing.

\section{Data Availability}

The authors declare that data supporting the findings of this study are available within the article.

\section{References}

1. Watson R, Crump H, Imison C, Currie C, Gaskins M. Emergency general surgery: challenges and opportunities. London, 2016.

2. National Emergency Laparotomy Audit. The second patient report of the National Emergency Laparotomy Audit (NELA) December 2014 to November 2015. London, 2016.

3. O'Connor DB, Winter DC. The role of laparoscopy in the management of acute small-bowel obstruction: a review of over 2,000 cases. Surg Endosc. 2012;26(1):12-17.

4. Ray NF, Denton WG, Thamer M, Henderson SC, Perry S. Abdominal adhesiolysis: inpatient care and expenditures in the United States in 1994. J Am Coll Surg. 1998;186(1):1-9.

5. Sajid MS, Khawaja AH, Sains P, Singh KK, Baig MK. A systematic review comparing laparoscopic vs open adhesiolysis in patients with adhesional small bowel obstruction. Am J Surg. 2016;212(1):138-150.

6. Bhangu A, Kolias AG, Pinkney T, Hall NJ, Fitzgerald JE. Surgical research collaboratives in the UK. Lancet. 2013;382(9898):1091-1092.

7. Lee MJ, Sayers AE, Wilson TR, Acheson AG, Anderson 
ID, Fearnhead NS, the NASBO Steering Group. Current management of small bowel obstruction in the UK: results from the National Audit of Small Bowel Obstruction clinical practice survey. Colorectal Dis. 2018;20(7):623630 .

8. Adams S, Wilson T, Brown AR. Laparoscopic management of acute small bowel obstruction. Aust N Z J Surg. 1993;63(1):39-41.

9. Bailey IS, Rhodes M, O'Rourke N, Nathanson L, Fielding G. Laparoscopic management of acute small bowel obstruction. Br J Surg. 1998;85(1):84-87.

10. Agresta F, Piazza A, Michelet I, Bedin N, Sartori CA. Small bowel obstruction. Laparoscopic approach. Surg Endosc. 2000;14(2):154-156.

11. Wullstein C, Gross E. Laparoscopic compared with conventional treatment of acute adhesive small bowel obstruction. Br J Surg. 2003;90(9):1147-1151.

12. Di Saverio S, Coccolini F, Galati M, Smerieri N, Biffl WL, Ansaloni L, Tugnoli G, et al. Bologna guidelines for diagnosis and management of adhesive small bowel obstruction (ASBO): 2013 update of the evidence-based guidelines from the world society of emergency surgery ASBO working group. World J Emerg Surg. 2013;8(1):42.

13. Catena F, Di Saverio S, Kelly MD, Biffl WL, Ansaloni L, Mandala V, Velmahos GC, et al. Bologna guidelines for diagnosis and management of Adhesive Small Bowel Obstruction (ASBO): 2010 evidence-based guidelines of the world society of emergency surgery. World J Emerg Surg. 2011;6:5.

14. Wiggins T, Markar SR, Harris A. Laparoscopic adhesiolysis for acute small bowel obstruction: systematic review and pooled analysis. Surg Endosc. 2015;29(12):34323442.

15. Mancini GJ, Petroski GF, Lin WC, Sporn E, Miedema BW, Thaler K. Nationwide impact of laparoscopic lysis of adhesions in the management of intestinal obstruction in the US. J Am Coll Surg. 2008;207(4):520-526.

16. Huang X, Fang G, Lin J, Xu K, Shi H, Zhuang L. A prediction model for recognizing strangulated small bowel obstruction. Gastroenterol Res Pract. 2018;2018:7164648.
17. Cevikel MH, Ozgun H, Boylu S, Demirkiran AE, Aydin N, Sari C, Erkus M. C-reactive protein may be a marker of bacterial translocation in experimental intestinal obstruction. ANZ J Surg. 2004;74(10):900-904.

18. Vilos GA, Ternamian A, Dempster J, Laberge PY, Clinical Practice Gynaecology C. Laparoscopic entry: a review of techniques, technologies, and complications. J Obstet Gynaecol Can. 2007;29(5):433-447.

19. Report of the National Audit of Small Bowel Obstruction 2017 (NASBO).

20. Sebastian-Valverde E, Poves I, Membrilla-Fernandez E, Pons-Fragero MJ, Grande L. The role of the laparoscopic approach in the surgical management of acute adhesive small bowel obstruction. BMC Surg. 2019;19(1):40.

21. Duepree HJ, Senagore AJ, Delaney CP, Fazio VW. Does means of access affect the incidence of small bowel obstruction and ventral hernia after bowel resection? Laparoscopy versus laparotomy. J Am Coll Surg. 2003;197(2):177-181.

22. Beck DE, Opelka FG, Bailey HR, Rauh SM, Pashos CL. Incidence of small-bowel obstruction and adhesiolysis after open colorectal and general surgery. Dis Colon Rectum. 1999;42(2):241-248.

23. Menzies D, Ellis H. Intestinal obstruction from adhesions-how big is the problem? Ann R Coll Surg Engl. 1990;72(1):60-63.

24. Burns EM, Currie A, Bottle A, Aylin P, Darzi A, Faiz O. Minimal-access colorectal surgery is associated with fewer adhesion-related admissions than open surgery. Br J Surg. 2013;100(1):152-159.

25. Reshef A, Hull TL, Kiran RP. Risk of adhesive obstruction after colorectal surgery: the benefits of the minimally invasive approach may extend well beyond the perioperative period. Surg Endosc. 2013;27(5):1717-1720.

26. Sallinen V, Di Saverio S, Haukijarvi E, Juusela R, Wikstrom H, Koivukangas V, Catena F, et al. Laparoscopic versus open adhesiolysis for adhesive small bowel obstruction (LASSO): an international, multicentre, randomised, open-label trial. Lancet Gastroenterol Hepatol. 2019;4(4):278-286. 